\title{
Erratum: Continuum interpretation of the dynamical-triangulation formulation of quantum Einstein gravity
}

\author{
Jan Smit \\ Institute for Theoretical Physics, University of Amsterdam, \\ Science Park 904, P.O. Box 94485, 1090 GL, Amsterdam, the Netherlands \\ E-mail: j.smit@uva.nl
}

ERRATUM TO: JHEP08(2013)016

ABSTRACT: An error in the numerical evaluation of the lattice-continuum conversion factor affects the magnitude of the continuum curvature in several plots. A corrected plot shows somewhat smaller systematic uncertainties. Another plot that would become less informative after correction is replaced by a more revealing one.

ARXiv EPRINT: 1304.6339

The continuum curvature $R_{\mathrm{c}}=R_{\mathrm{osc}} / \lambda^{2}= \pm 12 /\left(\lambda r_{0}\right)^{2}$ contains the factor $\lambda$ that converts the lattice geodesic-distance to the continuum distance. The DOB-fit $\lambda$-values 0.405 (0.465) quoted below (4.19) are wrong by a factor $\pi^{-1 / 4}$, due to an error in a Mathematica code. The correct values averaged over the nearly $\kappa_{2}$-independent values in the crumpled (elongated) phase are 0.3044 (0.3483). Consequently, in the plots depending on the DOBfit curvature, its $R_{\mathrm{c}}$ needs to be multiplied by a factor $\sqrt{\pi} \approx 1.8$. This affects figures ${ }^{1}$ 6, 15 and 21. To start with the latter, the corrected version below shows less systematic dependence on the fitting method in the elongated phase, since the DOB-fit $R_{\mathrm{c}}$ now lies in-between the $R_{\mathrm{c}}$ s of the $\mathrm{A}$-fit and B-fit.

The vertical scales in figure 6 and the right plot of figure 15 need to be multiplied by respectively $\sqrt{\pi}$ and $1 / \sqrt{\pi}$, which is easily visualized. However, because of the increased difference in magnitude between $R_{\mathrm{c}}$ and $R_{\mathrm{av}}-R_{\mathrm{s}}$, a corrected left plot in figure 15 would become unappealing to the eye and blur the difference in shape between $R_{\mathrm{c}}$ and $R_{\mathrm{av}}$. Below is an alternative plot showing $R_{\mathrm{c}}$ together with a renormalized Regge curvature defined as follows. The renormalizations $Z_{0}$ and $Z_{R}$ are chosen such that $R_{\mathrm{ren}}=\left(R_{\mathrm{av}}-Z_{0}\right) / Z_{R}$

\footnotetext{
${ }^{1}$ And the extrapolated $Z_{R}$ values $0.20(0.14)$ mentioned above figure 15 have to be divided by $\sqrt{\pi}$.
} 


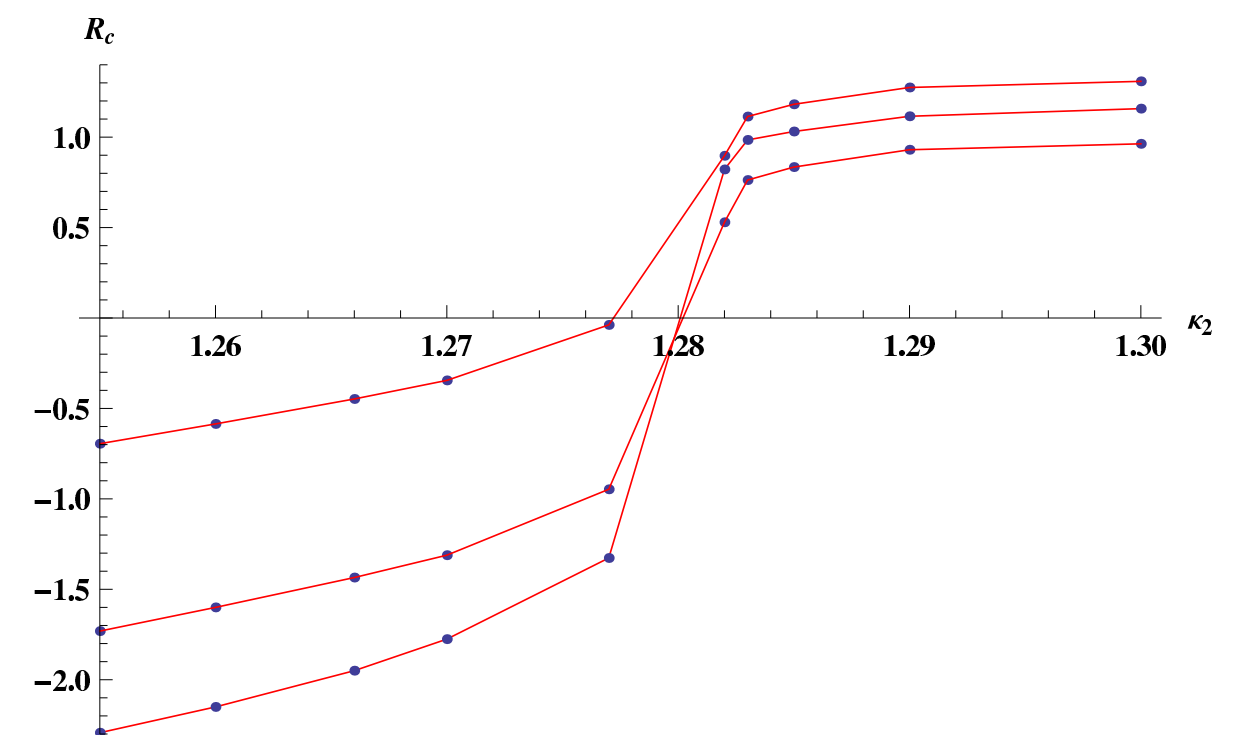

Figure 21. Curvatures $R_{\mathrm{c}}= \pm 12 /\left(\lambda r_{0}\right)^{2}$ with linear interpolation. From top to bottom in $\kappa_{2}>$ 1.28: A-fit, DOB-fit, B-fit.

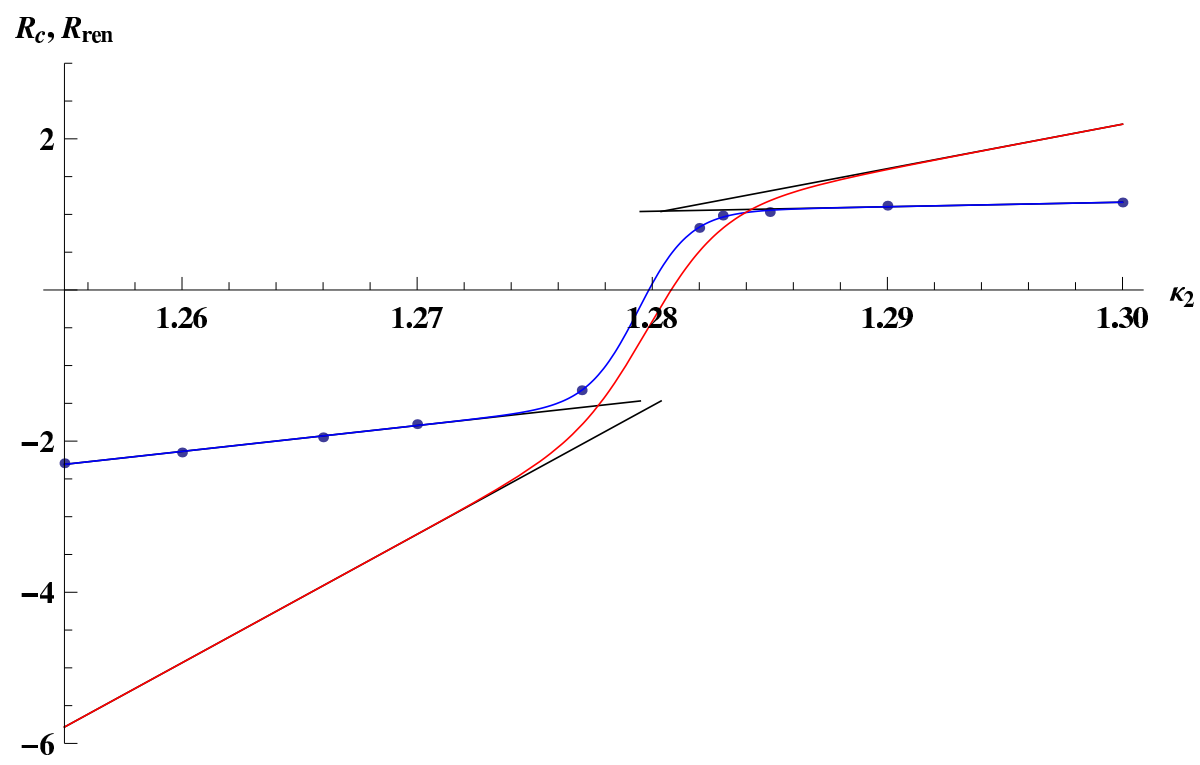

Figure 22. Alternative to a corrected left plot in figure 15. Data points of $R_{\mathrm{c}}$ (blue), its fit (8.6) (blue), $R_{\text {ren }}=\left(R_{\text {av }}-Z_{0}\right) / Z_{R}$ (red) with $R_{\text {av }}$ from the Gaussian-model fit, and extrapolations to their respective $\kappa_{2}^{\star}$ (black); $N_{4}=64000$, units $\tilde{\ell}=\ell / \sqrt{10}=1$.

matches $R_{\mathrm{c}}$ when extrapolated to the transition using their fitted functional forms. ${ }^{2}$ This means solving the two equations $\left(R_{s} \pm R_{\mathrm{d}}-Z_{0}\right) / Z_{R}=\left(R_{\mathrm{s}}^{\prime} \pm R_{\mathrm{d}}^{\prime}\right) / Z^{\prime}$ for $Z_{0}$ and $Z_{R}$ :

$$
Z_{0}=R_{\mathrm{s}}-R_{\mathrm{d}} \frac{R_{\mathrm{s}}^{\prime}}{R_{\mathrm{d}}^{\prime}} \simeq 7.0801 \tilde{\ell}^{-2}, \quad Z_{R}=\frac{R_{\mathrm{d}}}{R_{\mathrm{d}}^{\prime}} Z^{\prime} \simeq 0.0934
$$

${ }^{2}$ For the record: the fitted parameters of the fitting function (8.6) for $R_{\mathrm{c}}$ are $\tilde{\ell}^{2} R_{\mathrm{s}}^{\prime}=-0.02904, \tilde{\ell}^{2} R_{\mathrm{d}}^{\prime}=$ $0.1691, s_{\mathrm{s}}^{\prime}=148, s_{\mathrm{d}}^{\prime}=-104, Z^{\prime}=0.1349, \kappa_{2}^{\prime *}=1.27949$. 
Using these renormalizations ${ }^{3}$ the resulting $R_{\text {ren }}$ is plotted in figure 22 together with $R_{\mathrm{c}}$. The difference in slopes away from the transition is clear. In the elongated phase, the value of $R_{\mathrm{c}}$ extrapolated to the transition is, in the continuum interpretation, the curvature of an average branched-polymer component, a four-sphere of radius $r_{0 \mathrm{c}} \simeq 1.075 \ell$ and volume $\simeq 1510 v_{4}$.

Note added: the apparent approach to 1 of the alternative $Z_{R}$ based on a comparison of $R_{\text {av }}$ with the Gauss-Bonnet curvature of the total volume (suggestive of a higher than first-order transition) was a misleading clue, a red herring. The approximate volumeindependence of the parameters in table (8.4) (except $\kappa_{2}^{*}$ ) corroborates a first-order phase transition. Recently, strong evidence for a first-order transition was presented in [2].

Open Access. This article is distributed under the terms of the Creative Commons Attribution License (CC-BY 4.0), which permits any use, distribution and reproduction in any medium, provided the original author(s) and source are credited.

\section{References}

[1] J. Smit, Continuum interpretation of the dynamical-triangulation formulation of quantum Einstein gravity, JHEP 08 (2013) 016 [arXiv: 1304.6339] [INSPIRE].

[2] T. Rindlisbacher and P. de Forcrand, Euclidean dynamical triangulation revisited: is the phase transition really 1st order? (extended version), JHEP 05 (2015) 138 [arXiv: 1503.0370] [INSPIRE].

\footnotetext{
${ }^{3}$ The constant $Z_{R}$ is close to $\lambda^{2}$ averaged over the two phases. There appears to be no fundamental reason why this should be so. In two dimensions the conversion factor $\lambda$ for $R_{\mathrm{c}}$ is still nontrivial even in flat spacetime (as calculated in appendix A), whereas the volume-averaged Regge curvature needs no renormalization because $\int d^{2} x \sqrt{g} R$ is a topological invariant. In two dimensions, the Regge curvature will assign the same value as the Gauss-Bonnet curvature when an SDT configuration of spherical topology is viewed as the limit of a smooth volume-preserving deformation of a two-sphere.
} 